\title{
Recent results from COMPASS on the GPD program
}

\author{
Andrzej Sandacz ${ }^{1, *, *}$, on behalf of the COMPASS Collaboration \\ ${ }^{1}$ National Institute for Nuclear Research, Division of High Energy Physics, PL 00-681 Warsaw, Poland
}

\begin{abstract}
The dedicated COMPASS GPD program started in 2012 with a short pilot 'DVCS run' and the first results from this run on DVCS, exclusive $\pi^{0}$ and exclusive $\omega$ channels have been obtained. From the sum of cross sections measured with positive and negative beam polarities the DVCS cross-section and its dependence on the squared four-momentum transfer $t$ have been extracted. It allowed a first estimate of the transverse size of the partonic distribution of the nucleon $\sqrt{\left\langle r_{\perp}^{2}\right\rangle}$ in a range of intermediate $x_{B j}$ with a mean $x_{B j}$ value of 0.056 . We report also on the absolute value of the cross section for exclusive $\pi^{0}$ production and its dependence on $t$ and azimuthal angle $\phi$ between the scattering plane and $\pi^{0}$ production plane. These measurements are aiming to constrain the GPDs, in particular chiral-odd (transversity) GPDs. Further, we present results on the spin density matrix elements for exclusive $\omega$ meson production and discuss the contributions from unnaturaly parity exchanges and from transitions that do not conserve helicity.
\end{abstract}

\section{Introduction}

The theoretical framework of General Parton Distributions (GPDs) [1-3] provides a novel description of the nucleon's partonic structure and contains a wealth of new information. The GPDs provide a description of the nucleon as an extended object, sometimes referred to as a 3-dimensional 'nucleon tomography' [4], which correlates (transverse) spatial and (longitudinal) momentum degrees of freedom. The GPDs also allow access to such a fundamental property of the nucleon as the orbital angular momentum of quarks [2]. The mapping of the nucleon GPDs requires a comprehensive program of measuring various hard processes in a broad kinematic range, in particular Deeply Virtual Compton Scattering (DVCS). In addition, Hard Exclusive Meson Production (HEMP) is expected to add an independent and complementary information. A comprehensive experimental overview of DVCS is presented in Ref. [5] and results of a recent phenomenological studies and predictions for DVCS can be found in Ref. [6].

The GPD part of COMPASS-II proposal [7] is devoted to measurements of both DVCS and HEMP with polarised $\mu^{+}$and $\mu^{-}$beams and a liquid hydrogen target. The new detectors, the recoil proton detector CAMERA and the large-angle electromagnetic calorimeter ECAL0, which are essential for measurements of exclusive processes, were constructed and incorporated into the COMPASS set-up in 2012. The commissioning of these new detectors was done in 2012 and was followed by a 4-week pilot run. The results on DVCS, exclusive $\pi^{0}$

\footnotetext{
*Work supported by the Polish NCN Grant 2017/26/M/ST2/00498.

**e-mail: andrzej.sandacz@ncbj.gov.pl
} 
production and exclusive $\omega$ production, which are presented in this contribution, have been obtained from this pilot run.

\section{Experimental set-up}

COMPASS is a fixed-target experiment situated at the high-intensity M2 beam-line of the CERN SPS. It can deliver either hadron or naturally polarised muon beams of a given charge in the energy range between 50 and $280 \mathrm{GeV}$. For the GPD program the data are collected with the muon beam nominal energy of $160 \mathrm{GeV}$ and with muons of both polarities: $\mu^{+}\left(\mu^{-}\right)$ with polarisation of about $-0.8(+0.8)$.

The COMPASS apparatus [8] consists of a two-stage forward spectrometer comprising various tracking detectors, electromagnetic and hadronic calorimeters, muon identification detectors, and a ring imagining Cherenkov counter grouped around two dipole magnets SM1 and SM2. For the GPD program the set-up was complemented by installing the recoil proton detector CAMERA around the new $2.5 \mathrm{~m}$ long liquid hydrogen target and the large-angle electromagnetic calorimeter ECAL0. The 4-meter long CAMERA consists of two concentric barrels, each of 24 scintillator slats with a read out at both ends. The recoil proton detection is based on the ToF measurement between the two barrels. The calorimeter ECAL0 that is situated just downstream of CAMERA allows to extend the accessible kinematic domain for DVCS and exclusive $\pi^{0}$ production towards higher $x_{B j}$, improve hermeticity for detection of exclusive events and reduce the background to single-photon events production, which originates from $\pi^{0}$ and other decays. By adding these detectors COMPASS has been converted into a facility measuring exclusive reactions within kinematic domain from $x \sim 0.01$ to $\sim 0.15$, which cannot be explored at any other existing or planned facility in the near future.

\section{Results on DVCS}

The event selection exploits the overconstrained kinematics for the measured exclusive process $\mu p \rightarrow \mu^{\prime} p^{\prime} \gamma$. Events with a single outgoing muon and a single neutral cluster above the DVCS thresholds for the electromagnetic calorimeters are selected first. The muon and photon are then combined with all possible proton candidates reconstructed in CAMERA. The spectrometer information on incoming and scattered muons as well as on the positions and energy for the neutral cluster is used as a prediction to compare with kinematics of all track candidates that are found using the ToF system of the target recoil detector. Exemplary results of this comparison are displayed in Fig. 1 using two variables that characterize the kinematics of the recoiling target particle. Figure 1(left) shows the difference between the measured and the predicted azimuthal angle, $\Delta \phi$, and Fig. 1(right) the difference between the measured and the predicted transverse momentum, $\Delta p_{T}$. Here, $\phi$ and $p_{T}$ are given in the laboratory system. The combinatorial ambiguity is resolved by applying cuts on variables that are sensitive to the exclusivity.

The background is further reduced by applying a kinematic constrained fit. The input to the fit is supplied with all measured quantities, their uncertainties and correlations. The fit performs a chi-squared minimisation using constraints on the energy and momentum conservation. In the following the kinematic variables determined by the kinematic fit are used since the fit offers their most precise determination.

In this analysis we determine the differential cross section $\mathrm{d}^{4} \sigma^{\mu p \rightarrow \mu^{\prime} p^{\prime} \gamma} / \mathrm{d} Q^{2} \mathrm{~d} \nu \mathrm{d} t \mathrm{~d} \phi$ for exclusive muoproduction of single photon. Here, $Q^{2}$ and $v$ denote the virtual photon virtuality and its energy in the lab. system, $t$ is the square of the four-momentum transfer between the target and recoil protons and $\phi$ is the azimuthal angle between the lepton scattering plane and 

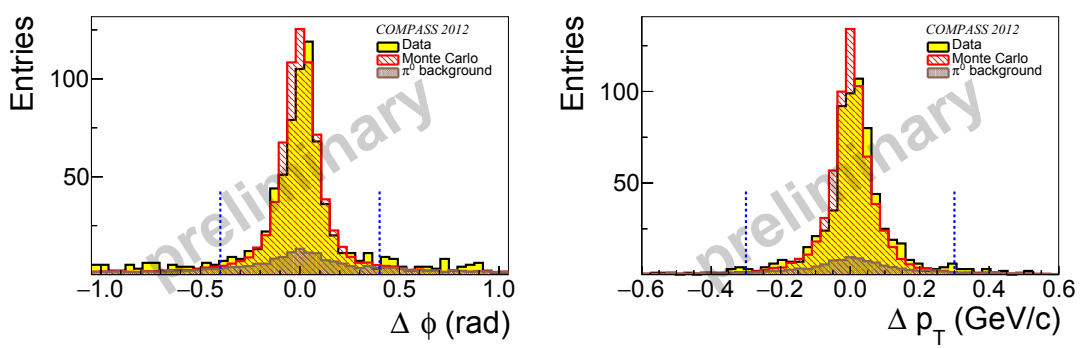

Figure 1: Distributions of the difference between predicted and reconstructed values of the recoiling proton candidates. The dashes blue vertical lines indicate the applied cuts. Here, Monte Carlo also includes $\pi^{0}$ background for the single photon sample.

the photon production plane. In order to extract the differential cross section as a function of $Q^{2}, v, t$ and $\phi$ the data has been corrected for acceptance effects using bin-wise acceptance correction factors.

This cross section was measured separately using either a $\mu^{+}$or $\mu^{-}$beam of $160 \mathrm{GeV} / c$ average momentum. The natural polarisation of the muon beam originates from the parityviolating deacy-in-flight of the parent meson, which implies opposite signs of the polarisation for the used $\mu^{+}$and $\mu^{-}$beams. Denoting charge and helicity of the incident muon by $+(-)$ and $\leftarrow(\rightarrow)$, the sum of the cross sections for $\mu^{+}$and $\mu^{-}$beams reads:

$$
2 \mathrm{~d} \sigma \equiv \mathrm{d} \sigma^{\stackrel{+}{\leftarrow}}+\mathrm{d} \sigma^{-}=2\left(\mathrm{~d} \sigma^{B H}+\mathrm{d} \sigma_{\text {unpol }}^{D V C S}-\left|P_{\mu}\right| \operatorname{Im} I\right),
$$

where $P_{\mu}$ denotes polarisation of the muon beam. The single-photon final state in leptonnucleon scattering can also originate from the $\mathrm{BH}$ process $(\mathrm{BH})$. Hence the DVCS and $\mathrm{BH}$ interfere, so that the above sum comprises also the contribution from the interference term denoted by $\operatorname{Im} I$.

After subtracting the exactly calculable BH cross section from Eq. (1) and integrating the remainder over $\phi$ there remains only the dominant contribution from transversely polarised virtual photons to the DVCS cross section $\mathrm{d}^{3} \sigma_{D V C S}^{\mu p \rightarrow \mu^{\prime} p^{\prime} \gamma} / \mathrm{d} Q^{2} \mathrm{~d} v \mathrm{~d} t$.

A major background source for single photon production is the production of $\pi^{0}$ mesons, where one of the decay photons is undetected. The contamination arising from $\pi^{0}$ production was estimated and subtracted from the data inside each bin using LEPTO 6.5.1 together with an exclusive $\pi^{0}$ Monte Carlo based on a parameterisation from Goloskokov-Kroll model [9].

The goal of this measurement is to determine the $t$-dependence of the unpolarised $(\phi$ integrated) DVCS cross section in virtual photon-proton scattering. The $\gamma^{*} p$ cross section is derived from the measured muon-proton cross section by taking into account the transverse virtual photon flux $\Gamma$

$$
\frac{\mathrm{d} \sigma^{\gamma^{*} p}}{\mathrm{~d} t}=\frac{1}{\Gamma\left(Q^{2}, v, E_{\mu}\right)} \frac{\mathrm{d}^{3} \sigma_{D V C S}^{\mu p}}{\mathrm{~d} Q^{2} \mathrm{~d} v \mathrm{~d} t},
$$

where according to the Hand's convention

$$
\Gamma\left(Q^{2}, v, E_{v}\right)=\frac{\alpha_{e m}\left(1-x_{B j}\right)}{2 \pi Q^{2} y E_{\mu}}\left[y^{2}\left(1-\frac{2 m_{\mu}^{2}}{Q^{2}}\right)+\frac{2}{1+Q^{2} / v^{2}}\left(1-y-\frac{Q^{2}}{4 E_{\mu}^{2}}\right)\right] .
$$

The values of the differential cross section $\mathrm{d} \sigma_{D V C S}^{\gamma^{*} p \rightarrow \gamma p} / \mathrm{d}|t|$ for four bins of $|t|$ are shown in 

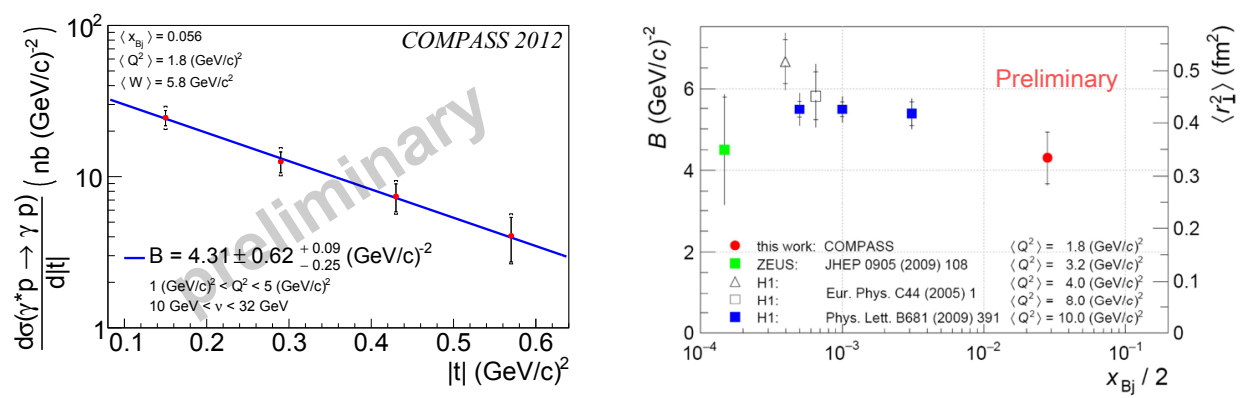

Figure 2: Left: The differential DVCS cross section as a function of $|t|$. Right: COMPASS results for the t-slope parameter compared to previous results from HERA.

Fig. 2 (left). They have been fitted with an exponential function in order to determine the $t$-slope parameter $B$. The value of $B$ is equal to $\left(4.3 \pm 0.6_{-0.3}^{+0.1}\right)(\mathrm{GeV} / c)^{-2}$ inside the kinematic range that is indicated in the legend of Fig. 2 (left), and which corresponds to $\left\langle x_{B j}\right\rangle=0.056$, $\left\langle Q^{2}\right\rangle=1.8(\mathrm{GeV} / c)^{2}$ and $\langle W\rangle=5.8 \mathrm{GeV}$. The extracted value of $B$ allows us to estimate the transverse size of partonic distibution in the nucleon $r_{\perp}$ using the relation

$$
\left\langle r_{\perp}^{2}\left(x_{B j}\right)\right\rangle=2 B\left(x_{B j}\right)
$$

which for our measurement results in $\sqrt{\left\langle r_{\perp}^{2}\right\rangle}=\left(0.58 \pm 0.04 \pm_{-0.02}^{+0.01}\right)$ fm at a mean $x_{B j}$ value of 0.056 .

The main systematic error of the cross section is due to uncertainties of absolute normalisation and determination of the amount of $\pi^{0}$ background. In case of parameter $B$ the error from the absolute normalisation plays a minor role, while the main systematic uncertainty originates from the normalisation of the $\pi^{0}$ background.

In Fig. 2 (right) the values of parameter $B$ from COMPASS and HERA [10,11] are plotted as a function of $x_{B j}$. As the slope $B$ is related to the transverse size of the partonic distribution (see Eq. (4)), one may conclude that these model-independent results indicate a decrease of the transverse size of the proton with increasing $x_{B j}$.

\section{Results on exclusive $\pi^{0}$ production}

For the selection of the sample of exclusive $\pi^{0}$ production, $\mu p \rightarrow \mu^{\prime} p^{\prime} \pi^{0}$, an analogous procedure is used as described for the DVCS sample. In addition to the cuts on exclusivity variables, a cut on the invariant mass of two-photon system is applied. In order to determine background originating from non-exclusive events, the data are modeled with Monte Carlo in both kinematic regions where either the signal or the background dominates. Here, LEPTO 6.5.1 is used for a non-exclusive part. For the signal contribution a dedicated Monte Carlo generator [12] was used with the cross section parameterisation from the Goloskokov-Kroll model. The background is subtracted bin-wise from the data. Other sources of background, like misidentified exclusive $\omega \rightarrow \pi^{0} \gamma \rightarrow \gamma \gamma \gamma$ events, where one photon is lost, are found to be negligible.

First, we extract the four-fold differential $\mu p$ cross sections separately for $\mu^{+}$and $\mu^{-}$data. Then the unpolarised cross section is obtained as the weighted mean of the two. In this way the dependence on the beam polarisation cancels. In order to convert $\mu p$ cross section into 
$\gamma^{*} p$ cross section we divide the former one by the tranasverse virtual photon flux (cf. Eq. (3)).

The systematic uncertainty in this measurement is mostly due to scaling, which can be decomposed into effects depending on the absolute normalisation and background estimate, as well as to the threshold effects and kinematic uncertainties.

The unpolarised $\gamma^{*} p$ cross section for exclusive $\pi^{0}$ production reads

$$
\frac{\mathrm{d}^{2} \sigma^{\gamma^{*} p}}{\mathrm{~d} t \mathrm{~d} \phi}=\frac{1}{2 \pi}\left[\frac{\mathrm{d} \sigma_{T}}{\mathrm{~d} t}+\epsilon \frac{\mathrm{d} \sigma_{L}}{\mathrm{~d} t}+\epsilon \cos (2 \phi) \frac{\mathrm{d} \sigma_{T T}}{\mathrm{~d} t}+\sqrt{2 \epsilon(1+\epsilon)} \cos (\phi) \frac{\mathrm{d} \sigma_{L T}}{\mathrm{~d} t}\right],
$$

where $\sigma_{T}, \sigma_{L}, \sigma_{T T}, \sigma_{L T}$ are the structure functions, $\epsilon$ is the virtual photon polarisation parameter and $\phi$ represents the azimuthal angle between lepton scattering plane and $\pi^{0}$ production plane. Here, the subscript $T(L)$ denotes the contribution from transversely (longitudinally) polarised virtual photons, while the subscripts $T T$ and $L T$ denote the contributions from the interference between transversely-transversely and longitudinally-transversely polarised photons.
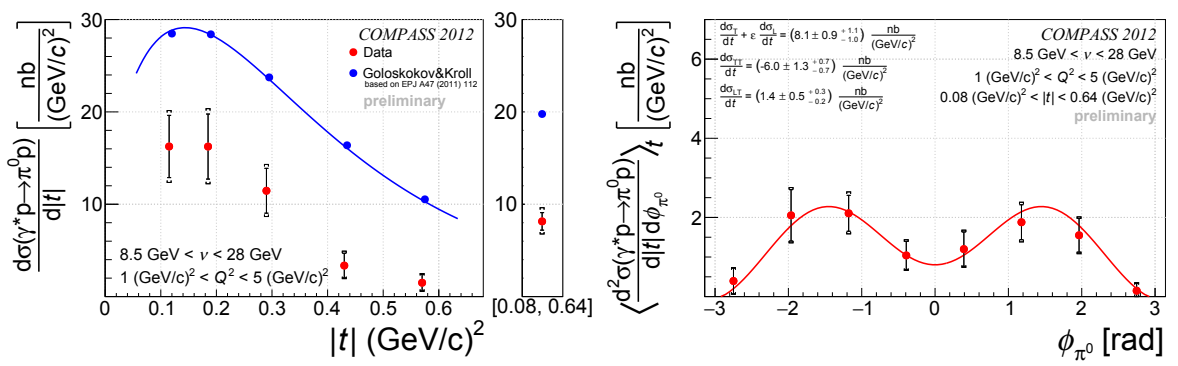

Figure 3: Left: The exclusive $\pi^{0}$ cross section as a function of $|t|$. COMPASS results are compared to predictions of the GPD model of Ref. [13]. Right: The exclusive $\pi^{0}$ cross section as a function of $\phi$. Solid line indicates the result of the fit with the values of the fitted parameters shown in the legend.

After integration over $\phi$, we extract the cross section in bins of $|t|$, which is displayed in Fig. 3 (left). It decreases exponentially with increasing $|t|$ at values of $|t|$ larger than about 0.25 $(\mathrm{GeV} / c)^{2}$, while at smaller $|t|$ the $t$-dependence becomes weaker. Being the first measurement at energies significantly higher than previous results, it is worth to compare it to the model prediction. The COMPASS result is compared to the prediction of the Goloskokov-Kroll model [13]. The blue curve represents the cross section from the model as a function of $t$, while the blue points correspond to the cross section averaged over $|t|$ bins of the data. The mean cross sections for the full $t$-range are compared at the rightmost part of the plot. We observe that the magnitude of the predicted cross section overshoots our measurement by approximately a factor of two.

Furthermore, we extract the cross section as a function of $\phi$ in the range of measured $t$ and eight bins of $\phi$ of equal width. The red dots in Fig. 3 (right) show the measured cross section for each bin and a fit of the function given by Eq. (5), which is represented by the red curve. The values of the fitted parameters are indicated in the legend of the plot. We observe a large contribution from $\sigma_{T T}$ and a smaller positive one from $\sigma_{L T}$. This indicates a significant role of transversely polarised photons in exclusive $\pi^{0}$ production.

According to Refs. $[13,14]$ the structure functions for exclusive pseudo-scalar meson production, which appear in Eq. (5), depend on GPDs $\tilde{H}^{q}, \tilde{E}^{q}, H_{T}^{q}$ and $\bar{E}_{T}^{q}=2 \tilde{H}_{T}^{q}+E_{T}^{q}$. For $\pi^{0}$ production a large contribution from transversely polarised virtual photons is expected, 
which is mainly generated by the chiral-odd ('transversity') GPDs $\bar{E}_{T}^{q}$. It manifests itself in a large contribution from $\sigma_{T T}$ and a dip in the differential cross section $\mathrm{d} \sigma / \mathrm{d} t$ as $|t|$ decreases to zero. These features are in a qualitative agreement with our results, although the predicted values of cross section overshoot the measured ones by a factor of about 2 .

\section{Results on exclusive $\omega$ production}

The considered process is $\mu p \rightarrow \mu^{\prime} p^{\prime} \omega$ with subsequent decays $\omega \rightarrow \pi^{+} \pi^{-} \pi^{0}$ and $\pi^{0} \rightarrow \gamma \gamma$. Therefore, the selected event should contain a track of scattered muon, two tracks of hadrons with opposite charges and two neutral clusters in an electromagnetic calorimeter. Because in this analysus the information from CAMERA is not used, in order to suppress non-exclusive background, the cut $\left|E_{\text {miss }}\right|<3.0 \mathrm{GeV}$ was applied, with $E_{\text {miss }}=\left(M_{X}^{2}-M_{p}^{2}\right) /\left(2 M_{p}\right)$. Here $M_{p}$ is the proton mass and $M_{X}^{2}=\left(p+q-p_{\pi^{+}}-p_{\pi^{-}}-p_{\pi^{0}}\right)^{2}$ is the missing mass squared, and $p, q$, $p_{\pi^{+}}, p_{\pi^{-}}$and $p_{\pi^{0}}$ are the four-momenta of target nucleon, virtual photon and each of the three pions.
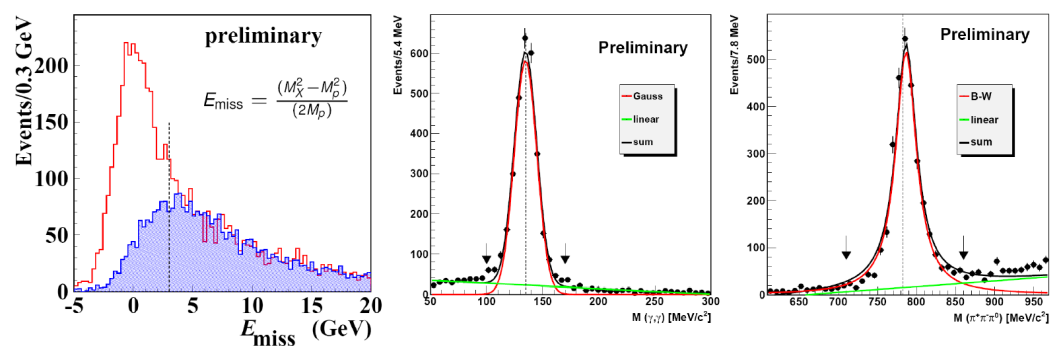

Figure 4: Left: $E_{\text {miss }}$ distribution. Shaded histogram corresponds to non-exclusive background estimated using LEPTO Monte Carlo. Middle: The $\gamma \gamma$ invariant mass distribution fitted by a sum of Gaussian function and a linear dependence for background. Right: The $\pi^{+} \pi^{-} \pi^{0}$ invariant mass fitted by a sum of Breit-Wigner function and a linear dependence for background. Dashed vertical lines denote the PDG values.

Selected kinematic distributions for the sample are shown in Fig. 4. These are $E_{\text {miss }}$ distribution, the invariant mass of two photons and the invariant mass of $\pi^{+} \pi^{-} \pi^{0}$ system. Clear $\pi^{0}$ and $\omega$ signals are observed. In the $E_{\text {miss }}$ distribution the data are shown as open histogram, while the shaded one corresponds to the background evaluated using LEPTO Monte Carlo. The amount of background in the signal window $\left|E_{\text {miss }}\right|<3.0 \mathrm{GeV}$ depends on $Q^{2}, W$ and $p_{T}^{2}$, and when averaged over the whole sample it amounts to $28 \%$.

The SDMEs describe the spin structure of the reaction. They are related to the bilinear combinations of helicity amplitudes that describe transitions between specified spin states of the virtual photon, target proton, produced vector meson and recoil proton. For an unpolarised nucleon target, after summing up over initial and final spin states of the proton, the SDMEs depend just on helicities of the virtual photon and produced meson. The values of SDMEs allow us to test the $s$-channel helicity conservation (SCHC) hypothesis, as well as to investigate the role of transversity (parton helicity-flip) GPDs and of unnatural parity exchange (UPE) mechanisms in exclusive $\omega$ production.

The SDMEs appear as parameters in the three dimensional angular distributions of the vector meson production and decay. Definitions of angles and reference frames are given e.g. in Ref. [15]. The SDMEs were determined by using the Unbinned Maximum Likelihood method to fit the experimental three-dimensional angular distribution of $\omega$ production and 
decay. The fitted distribution was a weighted superposition of the angular distributions for exclusive events and non-exclusive background. The fraction of background in the signal window was determined using $E_{\text {miss }}$ distribution as illustrated in Fig. 4.

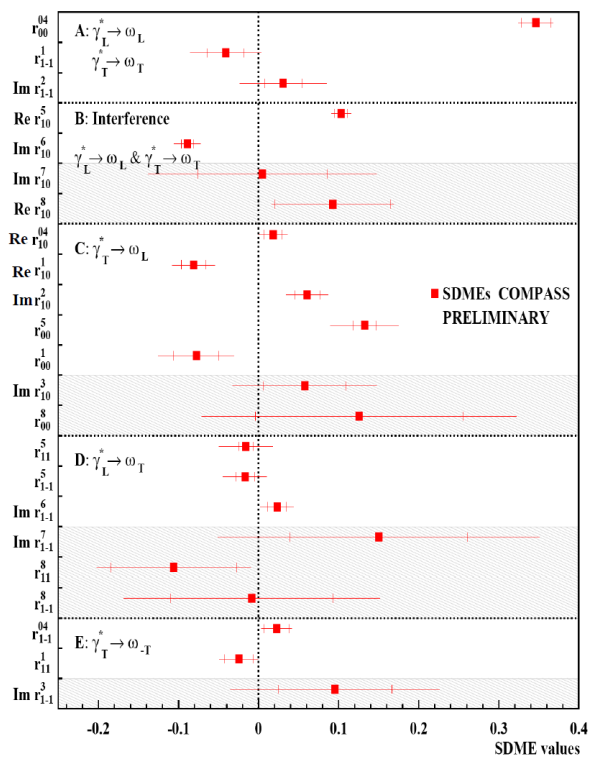

Figure 5: The 23 SDMEs for exclusive $\omega$ leptoproduction extracted in the entire COMPASS kinematic region. The inner error bars represent the statistical uncertainties, while the outer ones indicate the statistical and systematic uncertainties added in quadrature.

SDMEs for exclusive $\omega$ production extracted for the entire kinematic region are presented in Fig. 5. The average values of kinematic variables are $\left\langle Q^{2}\right\rangle=2.13(\mathrm{GeV} / c)^{2},\langle W\rangle=$ $7.6 \mathrm{GeV}$ and $\left\langle p_{T}^{2}\right\rangle=0.16(\mathrm{GeV} / c)^{2}$, where $p_{T}$ denotes the transverse momentum of $\omega$ meson relative to the direction of the virtual photon. The SDMEs are grouped in classes, each class corresponding to dominant transitions amplitudes with given helicities of the virtual photon and $\omega$ meson, as indicated in the figure.

In the case of SCHC, all SDMEs of classes $\mathrm{C}, \mathrm{D}$ and $\mathrm{E}$ should be equal to zero. However, for the transitions $\gamma_{T}^{*} \rightarrow V_{L}$ (class C) one observes a clear violation of SCHC. In the GK model [16] the SDMEs from this class are related to the contributions from transversity GPDs $\bar{E}_{T}$ and $H_{T}$ coupled with the higher-twist wave function of the meson. For instance the results for SDME $r_{00}^{5}$ are expected to be mostly affected by GPDs $\bar{E}_{T}$. Kinematic dependences of these SDMEs on $Q^{2}, W$, and $p_{T}^{2}$, which were also extracted in this analysis, may help to further constrain the model.

The existence of processes with unnatural parity exchange (UPE) in $\omega$ production on the proton can be tested by examining linear combination of SDMEs such as

$$
u_{1}=1-r_{00}^{04}+2 r_{1-1}^{04}-2 r_{11}^{1}-2 r_{1-1}^{1} .
$$

The quantity $u_{1}$ can be expressed in terms of helicity amplitudes and is proportional to the relative contribution of UPE processes to the cross section. Therefore, a different from zero value of $u_{1}$ would indicate a conribution of UPE processes. The kinematic dependence of $u_{1}$ on $Q^{2}, W$ and $p_{T}^{2}$ is shown in Fig.6. In the GK model the dominant UPE process is the pion 
pole exchange [17], which implies a decrease of $u_{1}$ with $W$. The data support the expected decrease.

\section{COMPASS preliminary}
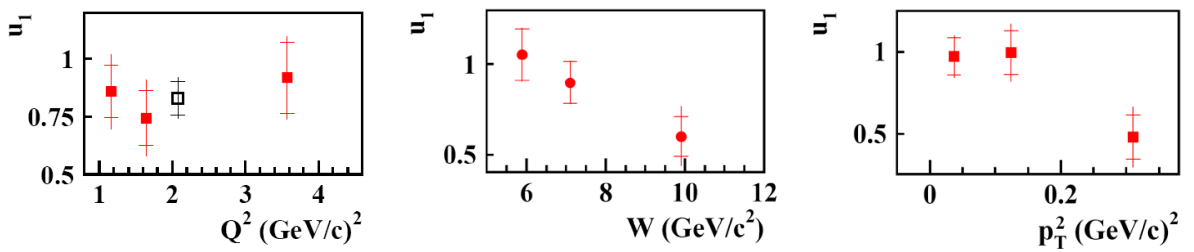

Figure 6: Dependence of quantity $u_{1}$ on kinematic variables $Q^{2}, W$ and $p_{T}^{2}$. The empty square respresents the $u 1$ value for the entire kinematic region.

\section{Outlook}

In 2016 and 2017 the COMPASS Collaboration has collected a large data sample on the GPD program. An expected increase of the statistic is by a factor of about 10 compared to the 2012 data. An investigation of GPDs with DVCS and HEMP on unpolarised protons using the data from 2016-2017 will allow us to determine the $x_{\mathrm{Bj}}$-dependence of $t$-slope of the differential cross sections. That is related to the spatial transverse distribution of partons and the 'nucleon tomography'. Measurements of the beam charge and spin sum and difference of single- $\gamma$ cross sections will give access to the real and imaginary parts of the DVCS amplitude, and will allow to further constrain GPDs $H$. Studies of exclusive production of vector mesons $\left(\rho^{0}, \omega, \phi\right)$ will lead to the quark flavour and gluon separation for GPDs $H$, while that of exclusive $\pi^{0}$ production will provide constraints on chiral-odd GPDs. The projected accuracies for measurements of various observables within the GPD program can be found in the COMPASS-II proposal [7].

\section{References}

[1] D. Mueller et al, Fortsch. Phys. 42 (1994) 101.

[2] X. Ji, Phys. Rev. Lett. 78 (1997) 610; Phys. Rev. D 55 (1997) 7114.

[3] A.V. Radyushkin, Phys. Lett. B 385 (1996) 333; Phys. Rev. D 56 (1997) 5524.

[4] M. Burkardt, Phys. Rev. D 62 (2000) 071503; erratum-ibid. D 66 (2002) 119903; Int. J. Mod. Phys. A 18 (2003) 173; Phys. Lett. B 595 (2004) 245.

[5] N. d'Hose, S. Niccolai and A. Rostomyan, Eur. Phys. J. A 52 (2016) 157.

[6] K, Kumerički, S. Liuti and H. Moutarde, Eur. Phys. J. A 52 (2016) 151.

[7] F. Gautheron et al. [COMPASS Collaboration], CERN-SPSC-2010-014, SPSC-P-340, May 2010.

[8] COMPASS Collaboration, P. Abbon et al., Nucl. Instr. Meth. A 577 (2007) 455.

[9] S.V. Goloskokov and P. Kroll, Eur. Phys. J. C 65 (2010) 137.

[10] ZEUS Collaboration, S. Chekanov et al., JHEP 0905 (2009) 108.

[11] H1 Collaboration, F.D. Aaron et al. Phys. Lett. B 681 (2009) 391; A. Aktas et al. Eur. Phys. J. C 44 (2005) 1.

[12] A. Sandacz and P. Sznajder, arXiv:1207.0333 [hep-ph].

[13] S.V. Goloskokov and P. Kroll, Eur. Phys. J. A 47 (2011) 112.

[14] CLAS Collaboration, 1. Bedlinsky et al., Phys. Rev. C 90 (2014) 025205.

[15] K. Schilling and G. Wolf, Nucl. Phys. B 61, 381 (1973).

[16] S.V. Goloskokov, P. Kroll, Eur. Phys. J. C 742725 (2014).

[17] S.V. Goloskokov, P. Kroll, Eur. Phys. J. A 50146 (2014). 